\title{
Enhancement of Chest Images by Automatic Adaptive Spatial Filtering
}

\author{
Miguel Souto, Jose Correa, Pablo G. Tahoces, Douglas Tucker, Katerina S. Malagari, Juan J. Vidal, \\ and Robert G. Fraser
}

\begin{abstract}
Postprocessing of the image data is an exciting capability of digital radiography that may improve diagnostic performance. We present a new algorithm that selectively enhances edges and contrast in both lungs and mediastinum while minimally amplifying noise in chest images. Using different size kernels, two smoothed images are generated from the original chest image. The two regions of interest (lungs and mediastinum) are identified based on the distribution of pixel values in the image. A modified nonlinear unsharp mask subtraction technique is then applied. The resulting image has enhanced high- and middle-frequency information in the mediastinum without distorting lung parenchyma or significantly enhancing noise. We consider that the technique employed in this study could be suitable for routine use although its true effectiveness in improving diagnostic accuracy awaits observerperformance evaluation that is currently under way. Copyright 1992 by W.B. Saunders Company
\end{abstract}

KEY WORDS: image processing, spatial frequency enhancement, digital radiography, chest radiography feature enhancement.

$\mathbf{P}$ OSTPROCESSING of image data is one of the capabilities of digital chest radiography that has been shown to contribute to the improvement of diagnostic performance..$^{1,2}$ Many processing methods have been investigated over the past years. ${ }^{3-9}$ Image processing algorithms can be applied either globally to the entire image or selectively to specific regions of the image. Controversy still exists about the utility of globally applied processing techniques in chest radiography. It has been shown that digital unsharp masking, a technique that supresses low frequencies while enhancing a selected range of high frequencies, results in an edgeenhanced image that can aid in the detection of mediastinal disease..$^{10}$ It has also been reported that globally applied high-frequency edge enhancement improves observer performance in the detection of interstitial lung disease ${ }^{11}$ and fine pulmonary lines ${ }^{12}$ in digital storage phosphor images. However, in other studies the processing degraded observer performance in identifying certain lung abnormalities by distorting the appearance of the pulmonary markings. ${ }^{13,14}$

In chest imaging, selective improvement of the mediastinum and retrocardiac areas is desired while maintaining minimal enhancement of pulmonary markings and rib trabecula. ${ }^{15,16}$ In comparison with global image processing, selective processing applies unique parameters to each different region of the image. Image segmentation is often achieved through a study of the image histogram. Recently, Sezan et al ${ }^{17}$ used anatomically selective unsharp masking that resulted in chest images with higher image quality than those obtained by nonselective unsharp masking. McAdams et al ${ }^{18}$ also proposed a technique for anatomically selective gray scale modification and unsharp masking in digital chest radiography. However, the method for determining the gray-level threshold requires human intervention and is therefore impractical for routine clinical application.

This report describes a new frequency processing algorithm that we have implemented and applied to chest radiographs. The resulting images are enhanced in both lungs and mediastinum, yet they maintain the appearance of the lungs similar to that of conventional radiographs.

\section{METHODS}

The images used in this study are standard adult chest radiographs $(35 \times 42 \mathrm{~cm})$ obtained from the radiology reading room at the University of Alabama. The films are digitized by a Konica laser film scanner (Konica Corp, Tokyo, Japan) at a resolution of 2,000 horizontal $\times 2,430$ vertical pixels $(2.86 \mathrm{cycles} / \mathrm{mm}$ horizontal and $2.89 \mathrm{cy} / \mathrm{mm}$ vertical). The optical density range from 0 to 4 is digitized to 10 bits of precision. Scanning requires 1 minute and 20 seconds per film. A DEC VAX computer (Digital Equipment Corp, Maynard, MA) running the VMS operating

From the Departments of Radiology of the Hospital General de Galicia, University of Santiago de Compostela, Spain; the University of Alabama at Birmingham Hospitals, USA; and the Evangelismos Hospital, Athens, Greece.

Supported by Fondo de Investigaciones Sanitarias de la Seguridad Social (Spain) grant no 91/0284.

Address reprint request to Robert G. Fraser, MD, Department of Radiology, University of Alabama at Birmingham, 619 S 19th Street, Birmingham, AL 35233.

Copyright 01992 by W.B. Saunders Company

0897-1889/92/0504-0008\$03.00/0 
system is used for all the calculations, the application programs being written in FORTRAN. Processing is performed on the image at full contrast and spatial resolution. Printed images are produced by an Agfa compact L laser printer (Agfa-Gevaert N.V., Mortsel, Beigium) interfaced to the VAX, and these films are photographed for publication. Care is taken in the photographic reproduction to ensure that all the images shown are acquired, developed and printed with the same techniques. Thus, any differences in detail can not be attributed to photographic processing. A functional block diagram of the system is shown in Fig 1.

The objective of spatial filtering is to enhance or supress specific spatial frequency components. Edge enhancement can be achieved by subtraction of a low-pass filtered image from the original image, and adding the resulting subtracted image back to the original image. This process is often called unsharp mask subtraction, and is expressed as:

$$
\begin{aligned}
I_{r}(x, y) & =I_{0}(x, y)+\left[K \times I_{h i}(x, y)\right], & & \text { (Equation 1A) } \\
I_{h i}(x, y) & =I_{0}(x, y)-I_{s}(x, y), & & \text { (Equation 1B) }
\end{aligned}
$$

where $I_{r}(x, y)$ is the resulting frequency enhanced image, $I_{o}(x, y)$ is the original image, $I_{h i}(x, y)$ and $I_{s}(x, y)$ are the highand low-(smoothed) frequency images, and $K$ is the weighting factor. The low-frequency smoothed image is formed by replacing each pixel value of the original image with the mean pixel value computed over a region centered at that pixel. The size of the area and the way in which neighboring pixels are used to produce the smoothed image defines the convolution kernel. ${ }^{19,20}$

The general principle of adaptive filtering has been described elsewhere. ${ }^{21}$ The technique uses the histogram of the image to determine the pixels (and regions) to which

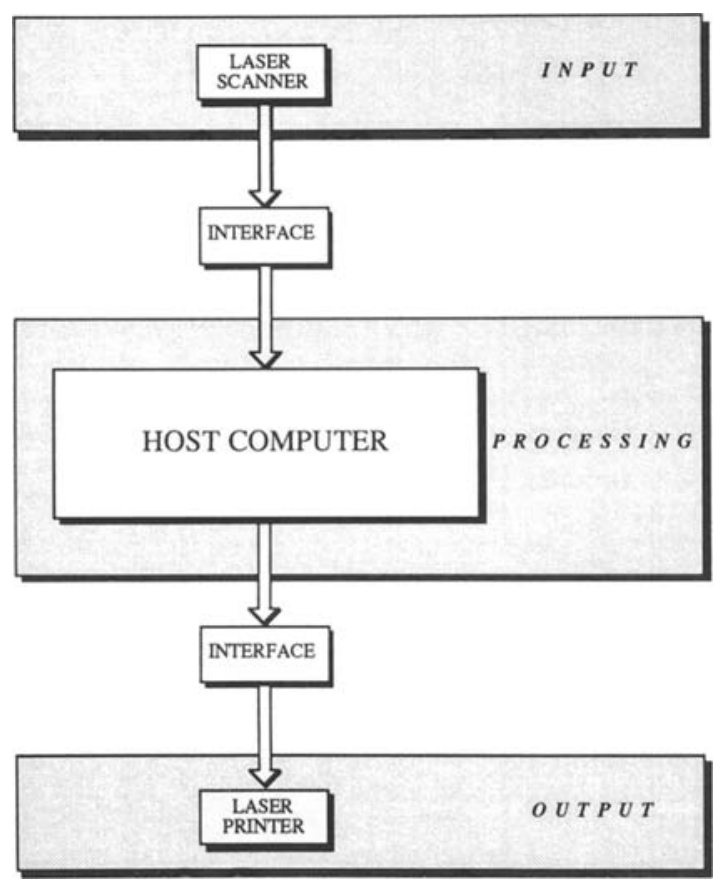

Fig 1. A functional block diagram of the system. enhancement is applied. This process allows filtering parameters be selectively applied to pixels of certain values. Our processing method is a three-step process that works as follows:

(1) Two low-frequency smoothed images, $\mathrm{I}_{\mathrm{s} 1}(\mathrm{x}, \mathrm{y})$, and $I_{s 2}(x, y)$, are generated by replacing the pixel value at the original image, $\mathrm{I}_{\mathrm{o}}(\mathrm{x}, \mathrm{y})$, with a mean pixel value computed over a square areas of $35 \times 35$ pixels, and $101 \times 101$ pixels.

(2) A modified unsharp mask subtraction is performed according to the following formulas:

$$
\mathrm{I}_{\mathrm{r}}(\mathrm{x}, \mathrm{y})=\mathrm{I}_{0}(\mathrm{x}, \mathrm{y})+\sum_{i=1,2} \mathrm{k}_{1}\left(\mathrm{I}_{0}(\mathrm{x}, \mathrm{y})\right) * \mathrm{I}_{\mathrm{hi}}(\mathrm{x}, \mathrm{y}), \quad \text { (Equation 2) }
$$

where

$$
\begin{array}{ll}
I_{h 1}(x, y)=I_{0}(x, y)-I_{s 1}(x, y), & \text { (Equation 3A) } \\
I_{h 2}(x, y)=I_{0}(x, y)-I_{s 2}(x, y), & \text { (Equation 3B) }
\end{array}
$$

and where $I_{r}(x, y), I_{o}(x, y)$ are as previously defined, $I_{s I}(x, y)$, $I_{s 2}(x, y)$, are the two smoothed images, $I_{h 1}(x, y)$ and $I_{h 2}(x, y)$ are the high-frequency images; $k_{1}$ and $k_{2}$ are weighting functions. $I_{r}(x, y)$ is an image in which the high- and medium-frequencies are selectively enhanced as a function of its grey level.

(3) Because $I_{r}(x, y)$ can have a very wide dynamic range, a nonlinear contrast enhancement function is automatically applied after filtering.

\section{RESULTS AND DISCUSSION}

Figure 2 is a laser printed unprocessed chest radiograph digitized at $2,000 \times 2,430 \times 10$ bits $(1,024$ gray levels). This image is used for all of the following processing routines. Figure 3 shows examples of frequency processing techniques that are applied globally to the original image shown in Fig 2. Figure 3A is the result of an unsharp mask subtraction as described in Equation 1 . The low-pass image is generated using a $35 \times 35$ pixel smoothing kernel and a constant weighting factor, $\mathrm{K}$, of 5 . In Fig 3B, two different sized smoothing kernels of $35 \times 35$ and $101 \times 101$ kernel sizes are used. The two smoothed images are linearly combined with the original to generate a high-frequency enhanced image. Constant weighting factors of 11 , -3 , and -5 are applied to the original $35 \times 35$ and $101 \times 101$ smoothed images, respectively. Figure 4 is a graphic representation of the frequency enhancement of the two images presented in Fig 3. The relative frequency enhancement (optical transfer factor) is plotted as a function of spatial frequency $(\mathrm{cy} / \mathrm{mm})$. The optical transfer factor is, for a specific frequency, the ratio of the frequency component in the enhanced image to that of the original image. Figure 4 shows that the second filter enhances lower frequency 


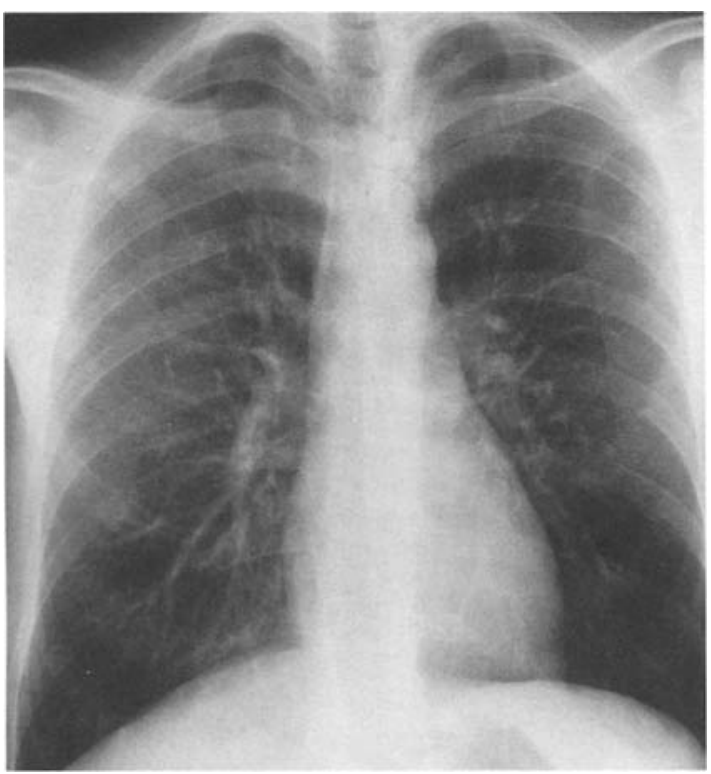

Fig 2. Original unprocessed digital chest image.

components than the first, whereas the first enhances higher frequencies more. Background noise typically possesses significant high-frequency components. The more the high-frequency component is amplified, the noisier the resultant image; as a result, Fig 3A appears noisier than Fig 3B. However, in both images,

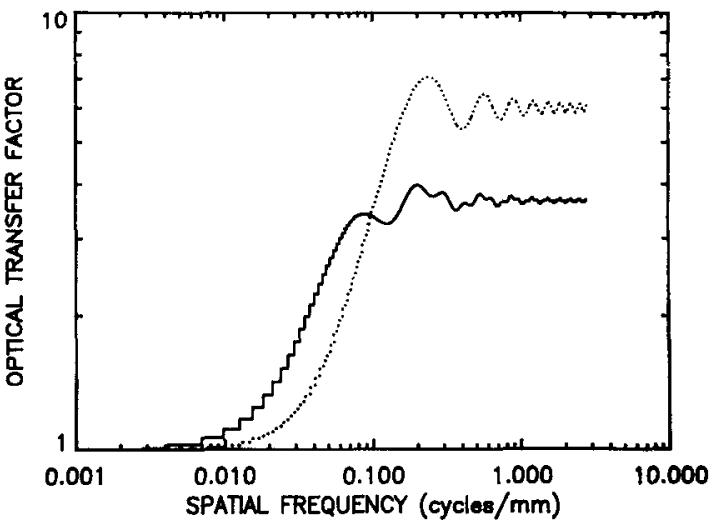

Fig 4. Optical transfer function for the two filters. The dotted curve is from Fig $3 A$. The solid curve is from Fig $3 B$.

normal pulmonary markings and trabecular pattern in the ribs are somewhat overprocessed, probably obscuring parenchymal detail.

Figure 5 represents the result of a modified nonlinear unsharp mask filter. As described previously, with this method a weighting function, dependent on the pixel value of the image, replaces the constant enhancement factor. Figure 5 is generated using the adaptive filtering process described above. The weighting factor is determined as a function of the original pixel value. Figure $6 \mathrm{~A}$ shows the weighting factor as a
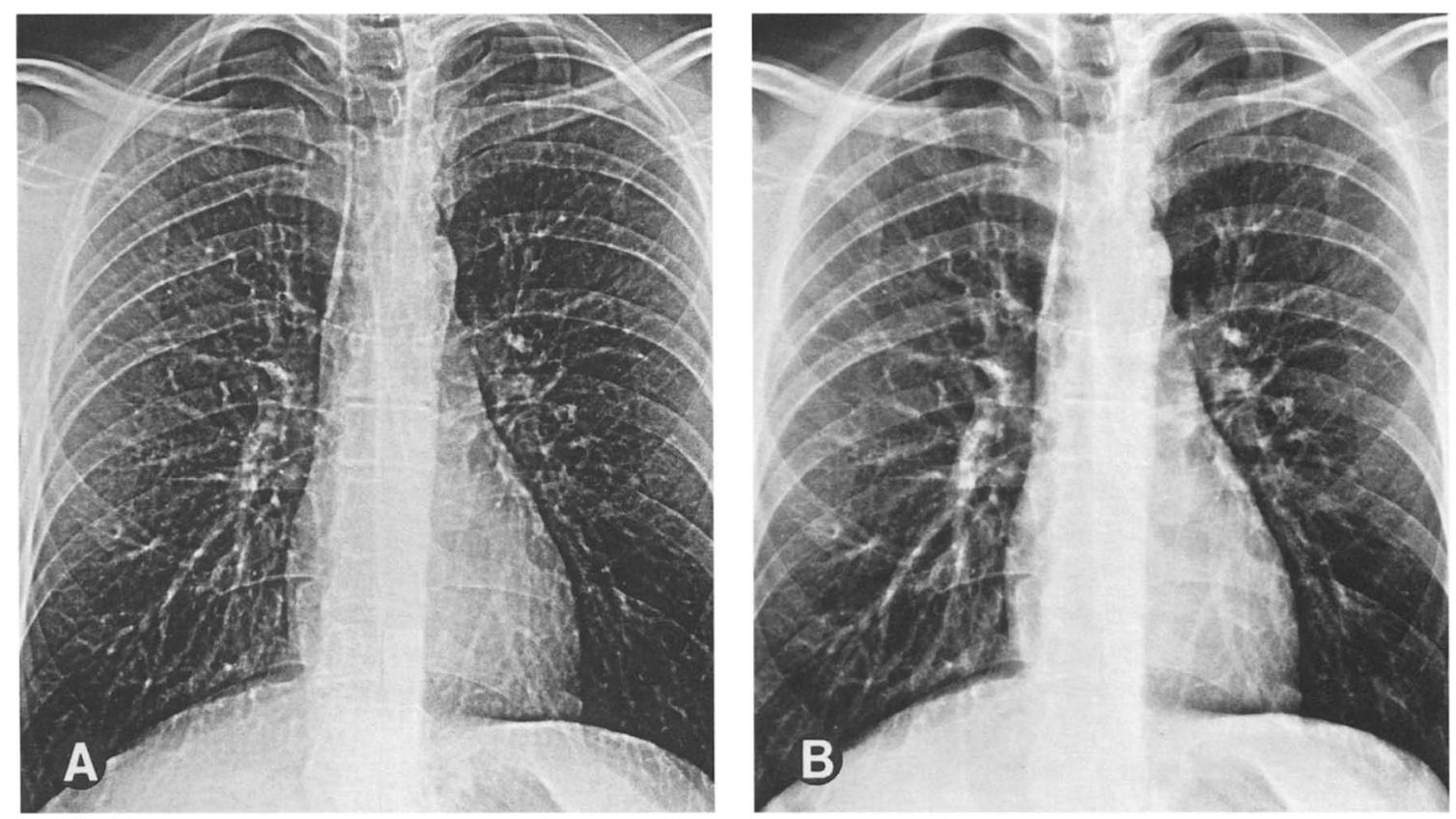

Fig 3. Globally processed images. (A) Using a kernel size of $35 \times 35$ pixels. (B) Using two different kernel sizes of $35 \times 35$ and $101 \times 101$ pixels. 


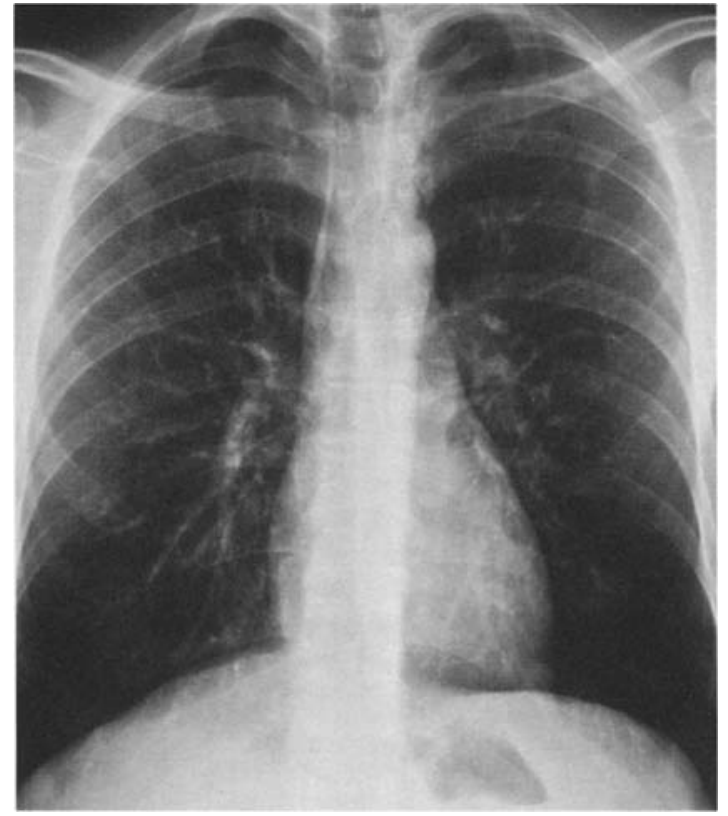

Fig 5. Adaptively processed image using two different smoothed images (kernel size $=35 \times 35$ and $101 \times 101$ pix. els.)

function of the pixel value used to generate Fig 5 . Figure $6 \mathrm{~B}$ is the histogram of the original chest image shown in Fig 2. One can observe from Fig 6 that the weighting factor is nearly constant at low- and high-pixel values, ie, lungs and mediastinum. In the lung region the value is nearly zero, whereas in the mediastinum the weighting reaches a maximum value. With this weighting, very little enhancement of the lung parenchyma occurs when the mediastinum is maximally enhanced. The transition between the minimum and maximum levels is smooth

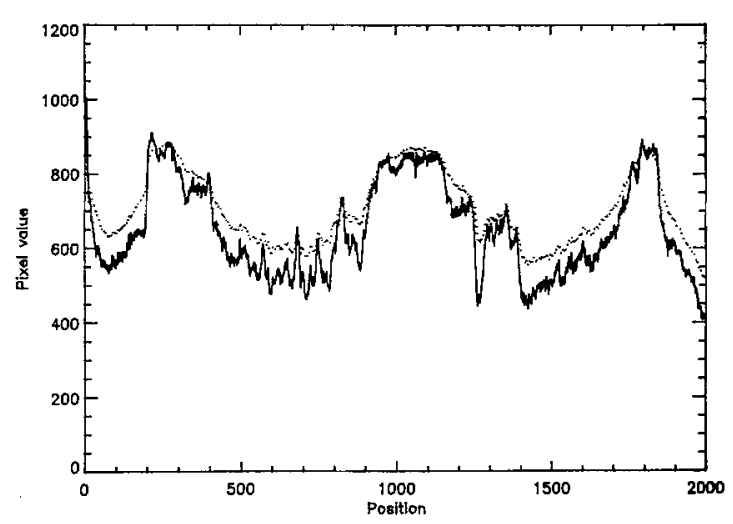

Fig 7. Line plot for a row across the central part of the chest radiograph. The dotted line is from Fig 2 . The solid line is from Fig 5B.

because sharp transitions cause artifacts at the mediastinum-lung interface. The role of the kernel size and its effect on image noise is similar to that of the globally applied unsharp mask subtraction. Lung markings are now satisfactorily enhanced; thus, the effect of minimizing image noise enhancement is clearly seen in Fig 5 in which the two smoothed images are generated. Details in the mediastinum (azygoesophageal recess, posterior and anterior junction lines, trachea, and bronchi), and retrocardiac area have been enhanced, while visibility of lung structures is adequate without distortion of pulmonary parenchymal detail. Clearer visualization of the effect of the selective processing of the algorithm is shown in Fig 7. This figure is a line plot (plot of pixel value versus position along one row of the image) through the central part of the original image and the adaptively
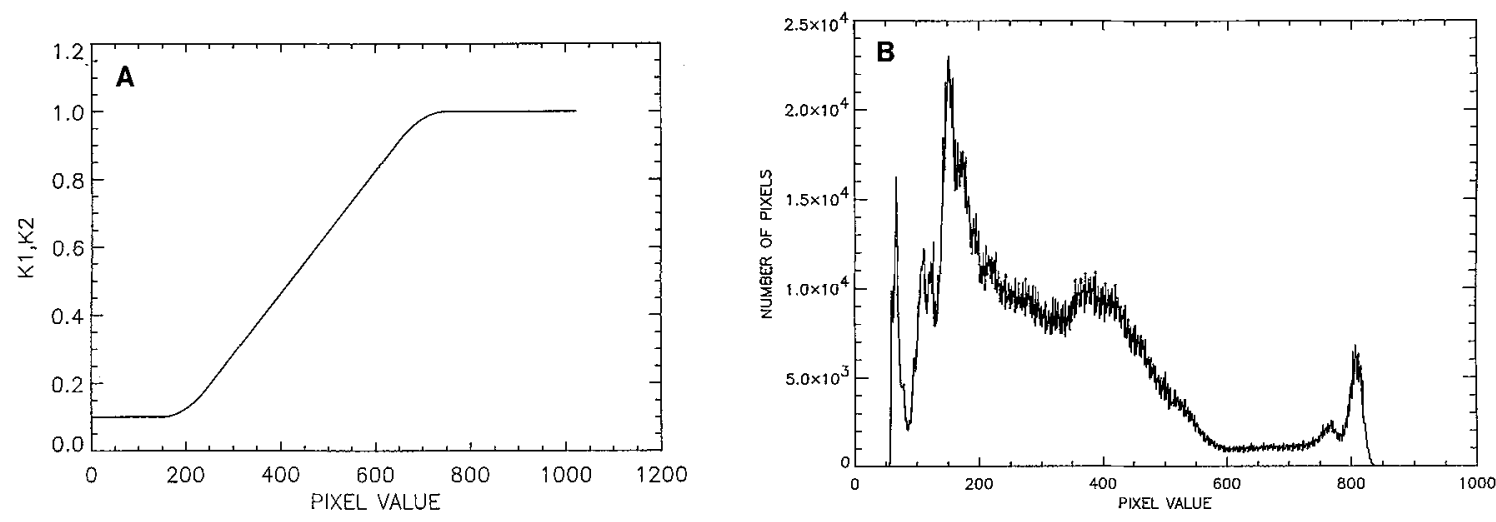

Fig 6. (A) Weighting factor ( $k$ ) curve as a function of the pixel values for the adaptive filter using the two different kernel sizes shown in Fig 5B. (B) Histogram of the digital chest image shown in Fig 2. 

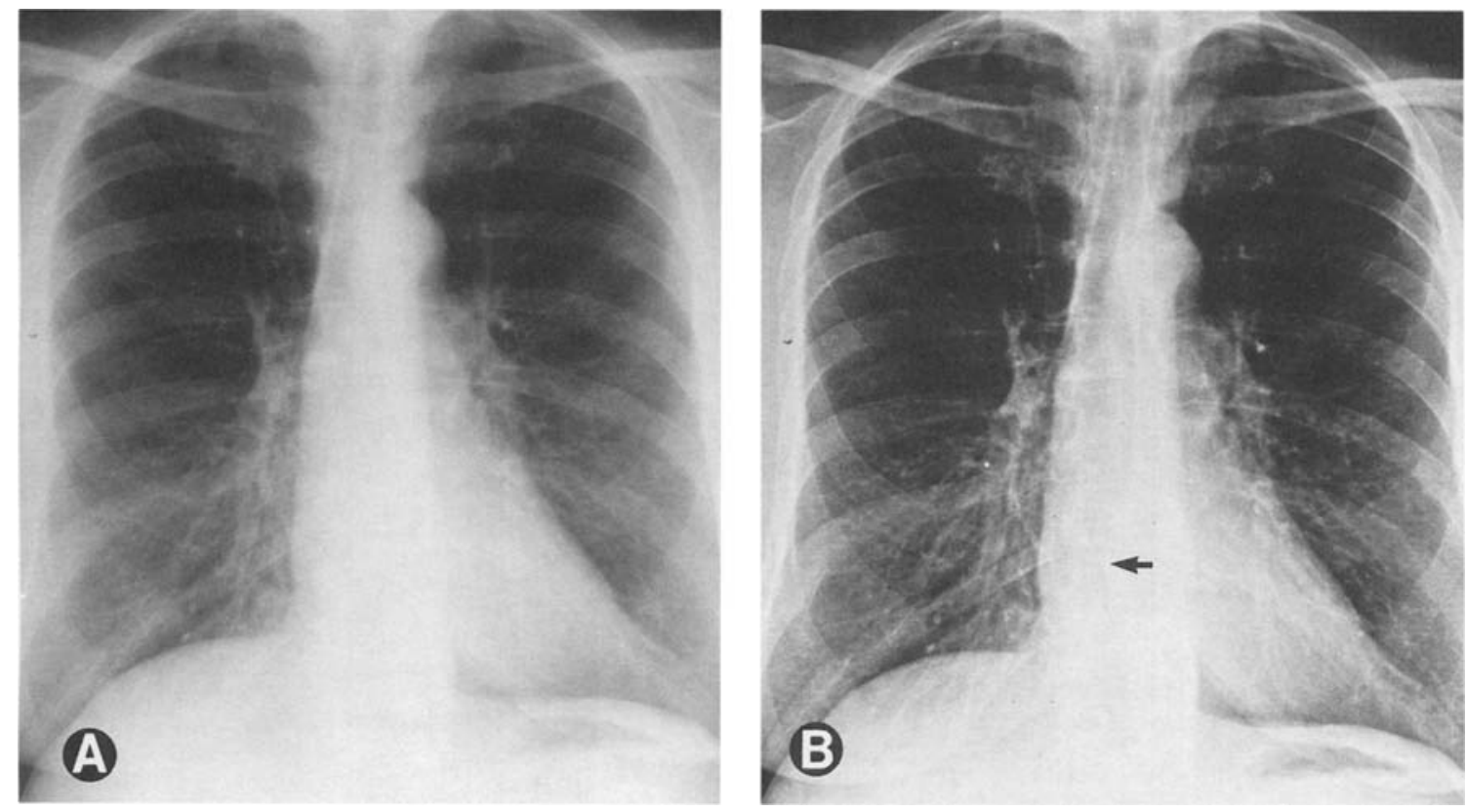

Fig 8. (A) Original unprocessed chest radiograph. (B) Same image after adaptive spatial filtering. Note the improved visibility of the local buldge of the azygoesophageal recess (arrow) caused by carcinoma of the esophagus.

filtered image. Over the lung, the curves show little difference, indicating that processing in these areas was mild. However, the middle zone of this line plot shows the enhancement of the structures in the mediastinum in the filtered image.

The role of the unsharp masking has been studied for several years. Unsharp mask subtraction technique can improve detectability of simple objects superimposed in radiographic noise if the smoothing kernel's size and weighting factor are properly selected. The kernel size determines the range of spatial frequencies to be enhanced in the processed image. If the kernel size is smaller, then the higher frequencies will be amplified. The frequencies selected by the kernel size are amplified proportionally by the weighting factor ${ }^{22-24}$ As shown in Fig 3A, the use of a small kernel size and a large weighting factor can decrease detectability because of increased high-frequency noise. The use of the two smoothed images obtained with different kernel sizes (one large and one small) when applied to a modified unsharp mask subtraction technique was investigated by Tahoces et al. ${ }^{25}$ After examining different combinations of kernel sizes, the two described here are considered appropriate for enhancing the spatial frequencies of interest while keeping image noise within clinically acceptable levels.

The use of the histogram-dependent weighting factor in unsharp mask filtering has been investigated by McAdams et al. ${ }^{26}$ They show that although the histogram of the entire chest image is influenced by extrathoracic structures, analysis of the chest cage alone shows a histogram with significant lung-mediastinum separation to establish a threshold. Their calculations indicated that the overlap of histogram regions between lungs and mediastinum is quite small and insensitive to clinical variables such as exposure or pathologic change. Our own studies based on 100 normal posteroanterior chest radiographs produced results in agreement with those of McAdams et al. ${ }^{27}$ The interface between lung and mediastinum was consistently within a small range of pixel values. This fact permits us to implement our adaptive filtering algorithm. The weighting function, $k$, has been developed empirically to provide maximum enhancement at the mediastinum without distortion of lung detail. Furthermore, the smooth transition between the minimum and maximum values produces the desired enhancement on the final image eliminating unwanted artifacts.

It has been shown that digital images and 
digital image processing have the potential to improve visual perception of diagnostically relevant information..$^{28}$ One of the questions that has important implications for digital imaging is the ability of the video display terminals (VDT) to display the same amount of diagnostic information that is available on hardcopy images. Several studies have found film to be decidely superior in diagnostic accuracy to a highresolution video display. ${ }^{29-32}$ However, it is possible that any technique that improves digital imaging systems might also improve observer performance using VDT images, thus widening their use in the medical arena. Computer time for our automatic adaptive spatial filtering is less than 3 minutes, a short enough period for routine clinical use. In addition, although three experienced chest radiologists participated in choosing the processing parameters, the efficacy of this technique compared with the globally applied algorithm and the original unprocessed images is currently being evaluated. A receiveroperating characteristic study using simulated (computer generated) solitary pulmonary nodules is in progress with the participation of the three departments that cooperated to produce the data for this paper. The results will be the subject of a future publication. Figures $8 \mathrm{~A}$ and $8 \mathrm{~B}$ show an example of the application of this technique in clinical practice.

\section{REFERENCES}

1. Barnes GT, Lauro K: Image processing in digital radiography: Basic concepts and applications. J Digit Imaging 2:132-146, 1989

2. Sherrier RH, Chotas HG, Johnson GA, et al: Image optimization in a computed-radiography/photostimulablephosphor system. J Digit Imaging 2:212-219, 1989

3. Templeton AW, Wetzel LH, Cook LT, et al: Enhancement of storage phosphor plate images: A C-language program. J Digit Imaging 5:59-63, 1992

4. Dobbins III JT, Powell AO: Variable compensation technique for digital radiography of the chest. Radiology 173:451-458, 1989

5. Sherrier RH, Johnson GA: Regionally adaptive histogram equalization of the chest. IEEE Trans Med Imaging 6:1-7, 1987

6. Daponte JS, Fox MD: Enhancement of chest radiographs with gradient operators. IEEE Trans Med Imaging 7:109-117, 1988

7. Rogowska J, Preston K, Sashin D: Evaluation of digital unsharp masking and local contrast stretching as applied to chest radiographs. IEEE Trans Biomed Eng 35:817-827, 1988

8. Chen C-C, Daponte JS, Fox MD: Fractal feature analysis and classification in medical imaging. IEEE Trans Med Imaging 8:133-142, 1989

9. Rehm K, Seeley GW, Dallas WJ, et al: Design and testing of artifact-supresses adaptive histogram equalization: A contrast-enhancement technique for display of digital chest radiographs. J Thorac Imaging 5:85-91, 1990

10. Schaefer CM, Greene R, Hall DA, et al: Mediastinal abnormalities: Detection with storage phosphor digital radiography. Radiology 178:169-173, 1991

11. Schaefer CM, Greene R, Llewellyn HJ, et al: Interstitial lung disease: Impact of postprocessing in digital storage phosphor imaging. Radiology 178:733-738, 1991

12. Oestman JW, Greene R, Rubens JR, et al: High frequency edge enhancement in the detection of fine pulmonary lines: Parity between storage phosphor digital images and conventional chest radiography. Invest Radiol 24:643-646, 1989

13. Goodman LR, Foley WD, Wilson CR, et al: Pneu- mothorax and other lung diseases: Effect of altered resolution and edge enhancement on diagnosis with digitized radiographs. Radiology 167:83-88, 1988

14. Oestman JW, Kushner DC, Bourgouin PM, et al: Subtle lung cancers: Impact of edge enhancement and gray scale reversal on detection with digitized chest radiographs. Radiology 167:657-658, 1988

15. Johnson GA, Danieley N, Ravin CE: Processing alternatives for digital chest imaging. Radiol Clin North Am 23:335-340, 1985

16. Sherrier RH, Chiles C, Wilkinson WE, et al: Effects of image processing on nodule detection rates in digitized chest radiographs: ROC study of observer performance Radiology 166:447-450, 1988

17. Sezan MI, Tekalp AM, Schaetzing R: Automatic anatomically selective image enhancement in digital chest radiography. IEEE Trans Med Imaging 8:154-162, 1989

18. McAdams HP, Johnson GA, Suddarth SA, et al Implementation of adaptive filtration for digital chest imaging. Optical Eng 26:669-673, 1987

19. Brody WR: Digital Radiography (ed 1). New York NY, Raven, 1984

20. Gonzalez RC, Wintz P: Digital Image Processing (ed 2) Reading, MA, Addison-Wesley 1987

21. Peli T, Lim JS: Adaptive filtering for image enhancement. Optical Eng 21:108-112, 1982

22. Giger ML, Doi K: Investigation of basic imaging properties in digital radiography. 1. Modulation transfer function. Med Phys 11:287-295, 1984

23. Giger ML, Doi K, Metz CE: Investigation of basic imaging properties in digital radiography. 2. Noise Wiener spectrum. Med Phys 11:797-805, 1984

24. Loo L-ND, Doi K, Metz CE: Investigation of basic imaging properties in digital radiography. 4. Effect of unsharp masking on the detectability of simple patterns. Med Phys 12:209-214, 1984

25. Tahoces PG, Correa J, Souto M, et al: Enhancement of chest and breast radiographs by automatic spatial filtering. IEEE Trans Med Imaging 10:330-335, 1991

26. MacAdams HP, Johnson GA, Suddarth SA, et al: 
Histogram-directed processing of digital chest images. Invest Radiol 21:253-259, 1986

27. Vidal JJ, Souto M, Tahoces PG, et al: Introduccion a la radiografia digital. Radiologia 33:231-239, 1991

28. Fraser RG, Sanders C, Barnes GT, et al: Digital imaging of the chest. Radiology 171:297-307, 1989

29. MacMahon H, Metz CE, Doi K, et al: Digital chest radiography: Effect on diagnostic accuracy of hard copy, conventional video, and reversed gray scale video display formats. Radiology 168:669-673, 1988

30. Slasky BS, Gur D, Good WF, et al: Receiver operat- ing characteristic analysis of chest image interpretation with conventional, laser-printed, and high-resolution workstation images. Radiology 174:775-780, 1990

31. Cox GG, Cook LT, McMiltan JH, et al: Chest radiography: Comparison of high-resolution digital displays with conventional and digital film. Radiology 176:771-776, 1990

32. Elam EA, Rehm K, Hillman BJ, et al: Efficacy of digital radiography for the detection of pneumothorax: Comparison with conventional chest radiography. AJR Am J Roentgenol 158:509-514, 1992 